The Astrophysical JOURnAL, 691, 2009 JANUARY 20, IN PRESS

Preprint typeset using LTEX style emulateapj v. 03/07/07

\title{
ION TEMPERATURES IN THE LOW SOLAR CORONA: POLAR CORONAL HOLES AT SOLAR MINIMUM
}

\author{
E. LANDI \\ Artep, Inc. at Naval Research Laboratory, 4555 Overlook Ave. S.W., 20375-5320, Washington DC
}

S. R. CRANMER

Harvard-Smithsonian Center for Astrophysics, 60 Garden Street, Cambridge, MA 02138

Draft version November 10, 2018

\begin{abstract}
In the present work we use a deep-exposure spectrum taken by the SUMER spectrometer in a polar coronal hole in 1996 to measure the ion temperatures of a large number of ions at many different heights above the limb between 0.03 and 0.17 solar radii. We find that the measured ion temperatures are almost always larger than the electron temperatures and exhibit a non-monotonic dependence on the charge-to-mass ratio. We use these measurements to provide empirical constraints to a theoretical model of ion heating and acceleration based on gradually replenished ion-cyclotron waves. We compare the wave power required to heat the ions to the observed levels to a prediction based on a model of anisotropic magnetohydrodynamic turbulence. We find that the empirical heating model and the turbulent cascade model agree with one another, and explain the measured ion temperatures, for charge-to-mass ratios smaller than about 0.25 . However, ions with charge-tomass ratios exceeding 0.25 disagree with the model; the wave power they require to be heated to the measured ion temperatures shows an increase with charge-to-mass ratio (i.e., with increasing frequency) that cannot be explained by a traditional cascade model. We discuss possible additional processes that might be responsible for the inferred surplus of wave power.

Subject headings: line: profiles — Sun: corona — Sun: UV radiation — techniques: spectroscopic — turbulence - waves
\end{abstract}

\section{INTRODUCTION}

In order to understand the physical processes that heat the solar corona and accelerate the solar wind, theories must be able to predict measurements of the plasma parameters in the regions that are being heated and accelerated. In the lowdensity, open-field regions that correspond to polar coronal holes on the Sun, the plasma is expected to become collisionless very near the solar surface, and thus the individual particle species (e.g., protons, electrons, and heavy ions) may exhibit diverging properties. These differences are key probes of the microscopic kinetic physics that drives the heating and acceleration. The Solar Ultraviolet Measurements of Emitted Radiation (SUMER) spectrometer aboard the Solar and Heliospheric Observatory ( $\mathrm{SOHO}$ ) has measured these properties for more than a decade (see, e.g., Wilhelm et al. 1995, 1997).

Inspired by both the SUMER observations presented below and other remote-sensing and in situ measurements, we focus on the damping of ion cyclotron resonant Alfvén waves as a natural means of preferentially heating and accelerating coronal ions. Efficient kinetic interactions between ion cyclotron waves and particle velocity distributions in the solar wind have been studied for several decades (e.g., AbrahamShrauner \& Feldman 1977; Hollweg \& Turner 1978; Marsch et al. 1982; Hollweg 1986; Tu \& Marsch 1997; Cranmer et al. 1999a; Hollweg \& Isenberg 2002; Cranmer 2002; Kohl et al. 2006). Despite much intense theoretical work, the origin of the high-frequency $\left(10^{2}-10^{4} \mathrm{~Hz}\right)$ cyclotron waves in the solar corona is not yet known. Most ideas for the generation of these waves fall into two classes: (1) excitation at the base of the corona, and (2) gradual growth or replenishment of cyclotron waves throughout the corona and solar wind. The first idea has been proposed to occur from impulsive processes taking place in magnetically complex regions (e.g., reconnection in microflares; see Axford \& McKenzie 1992; Ruzmaikin $\&$ Berger 1998). The second scenario has been proposed to occur mainly from magnetohydrodynamic (MHD) turbulent cascade (Isenberg \& Hollweg 1983; Cranmer \& van Ballegooijen 2003; Chandran 2005), but other processes such as kinetic microinstabilities have also been suggested. Indirect support for the second idea of "gradual replenishment" has come from empirical studies of radio scintillations (Hollweg 2000) and theoretical models of the charge and mass dependence of the wave damping (Cranmer 2000, 2001). In our paper, we will base our model on the gradual replenishment scenario.

One of the main predictions of ion-cyclotron models is preferential heating and acceleration of ions in the solar wind, with the effect of waves on ions being dependent on the $Z_{i} / A_{i}$ ratio, where $Z_{i}$ is the ion's charge and $A_{i}$ its atomic mass (with both usually specified in proton units). One of the observables that can best constrain theoretical models of ion-cyclotron waves is the behavior of the ion temperature (i.e., the magnitude of ion random motions on microscopic scales). These models are also dependent on the overall amplitude of MHD waves in the corona, which can also be constrained empirically by the so-called non-thermal component of emission line profiles.

Measuring ion temperatures and non-thermal velocities is however a very difficult task, as these are two separate physical quantities that have to be determined from one single observable, the line width. Thus, some assumptions have to be made. However, these limit the accuracy of the measurements. In the past, most assumptions were taken on the value of $T_{i}$, since the quantity of interest was the non-thermal velocity $v_{\text {nth }}$. The most common assumptions were $T_{i}=T_{e}$, or $T_{i}=T_{M}$, where $T_{M}$ is the temperature of formation of the 
line, usually taken as the temperature of maximum abundance of the ion emitting the line. A more elaborate set of assumptions were adopted by Seely et al. (1997), who assumed that ions formed in the same temperature range have the same $T_{i}$; this allowed them to determine both $T_{i}$ and $v_{\text {nth }}$ from SUMER/SOHO observations. Tu et al. (1998), on the contrary, assumed that $v_{\text {nth }}$ is the same for all ions. This allowed them to set an upper limit to $v_{n t h}$, and conservatively assumed $v_{\text {nth }}=0$ as a lower limit. From these limits, the range of possible $T_{i}$ values could be determined. In doing this, $\mathrm{Tu}$ et al. (1998) limited the number of assumptions to the minimum and chose not to determine $v_{\text {nth }}$, so that they could set rather precise limits to $T_{i}$ of the ions in their dataset. Dolla \& Solomon (2008) went a step further by making assumptions not on the values of $T_{i}$ or $v_{\text {nth }}$, but on the physical process that affects them. They assumed that: (1) non-thermal velocities are due to undamped Alfvén waves, and (2) that $\mathrm{Mg} \mathrm{X}$ was not heated by them so that its $T_{i}$ was constant and could be used to measure $v_{\text {nth }}$.

The aim of the present work is twofold. First, we will extend the analysis done by Tu et al. (1998) by applying their method to spectra emitted by coronal hole plasmas at the north pole, that include many more lines from many more ions than considered by them. We will repeat the $T_{i}$ measurements for as many heights above the solar limb as made possible by the signal-to-noise ratio and instrument scattered light. This has been achieved by using deep-exposure SUMER spectra encompassing the entire wavelength range (500-1500 ̊) included by detector B on SUMER.

Second, we will use the measured ion temperature values as constraints for semi-empirical theoretical models of ion heating and acceleration based on the damping of ion cyclotron resonant Alfvén waves. We will develop a model that is mainly intended to be illustrative of the proper ranges of plasma parameters that are consistent with the observations in the low corona, but not yet self-consistent enough to be considered a definitive or unique explanation of the SUMER data set. ${ }^{1}$

The first part of the paper deals with the ion temperature measurements. Section 2 introduces the method of analysis and Section 3 describes the observations studied in the present work. Our measurements are reported in Section 4 The second part of the paper describes the comparison with the theoretical model. In Section 5 the separation of line widths into thermal and non-thermal parts is discussed in more detail. The physical processes assumed in the semi-empirical models are given in Section 6, and a comparison with a purely theoretical model of MHD turbulence is made in Section 7] The model results are shown and described in Section 8 . Section 9 summarizes the present work.

\section{METHOD OF ANALYSIS}

In this work, we have applied the analysis method described by $\mathrm{Tu}$ et al. (1998), that we briefly summarize here. The measured full width half maximum (FWHM) $d \lambda$ of a spectral line can be related to the dynamical status of the emitting ion through the equation

$$
d \lambda=\frac{\lambda_{0}}{c} \sqrt{4 \ln 2\left(\frac{2 k_{\mathrm{B}} T_{i}}{M_{i}}+v_{\mathrm{nth}}^{2}\right)}
$$

\footnotetext{
${ }^{1}$ Specifically, these models do not try to validate or disprove the assumption that the ion cyclotron waves are created by an anisotropic turbulent cascade; they only try to work out the implications of such an assumption.
}

where $d \lambda$ is the FWHM of the line after the instrumental broadening has been removed, $T_{i}$ and $M_{i}=m_{p} A_{i}\left(m_{p}\right.$ is the proton mass) are the ion's temperature and mass, $k_{\mathrm{B}}$ is Boltzmann's constant, $c$ is the speed of light, $\lambda_{0}$ is the rest wavelength of the line, and $v_{\text {nth }}$ is the ion's non-thermal velocity. From equation (1), the ion temperature given by each line can not be larger than the value obtained when $v_{\text {nth }}=0$ :

$$
\begin{aligned}
T_{i} & \leq T_{\max , i} \\
T_{\max , i} & =\frac{m_{p} c^{2}}{8 k_{\mathrm{B}} \ln 2} A_{i}\left(\frac{d \lambda}{\lambda_{0}}\right)_{i}^{2}=1.96 \times 10^{12} A_{i}\left(\frac{d \lambda}{\lambda_{0}}\right)_{i}^{2} \mathrm{~K}
\end{aligned}
$$

where $A_{i}$ is the atomic mass. In the same way, the non-thermal velocity can not be larger than the value obtained when $T_{i}=0$ :

$$
\begin{aligned}
v_{\text {nth }} & \leq v_{\max , i} \\
v_{\max , i} & =\sqrt{\frac{c^{2}}{4 \ln 2}\left(\frac{d \lambda}{\lambda_{0}}\right)_{i}^{2}}=1.80 \times 10^{10}\left(\frac{d \lambda}{\lambda_{0}}\right)_{i} \mathrm{~cm} \mathrm{~s}^{-1} .
\end{aligned}
$$

In order to determine a lower limit to $T_{i}$, it is necessary to make an assumption on $v_{\text {nth }}$. The method devised by Tu et al. (1998) allows us to determine the minimum $T_{i}$ values compatible with a set of measured FWHM values by assuming that $v_{\text {nth }}$ is the same for all ions. If we define $v_{\min }$ as the smallest among the $v_{\max , i}$ values obtained from a set of lines, the ion temperature of each ion must be larger than the value obtained when $v_{\text {nth }}=v_{\min }$. If we indicate with $j$ the line for which $v_{\text {nth }}=v_{\text {min }}$, the range of ion temperatures compatible with the FWHM measured with line $i$ is given by

$$
\frac{m_{p} c^{2}}{8 k_{\mathrm{B}} \ln 2} A_{i}\left[\left(\frac{d \lambda}{\lambda_{0}}\right)_{i}^{2}-\left(\frac{d \lambda}{\lambda_{0}}\right)_{j}^{2}\right] \leq T_{i} \leq \frac{m_{p} c^{2}}{8 k_{\mathrm{B}} \ln 2} A_{i}\left(\frac{d \lambda}{\lambda_{0}}\right)_{i}^{2}
$$

Note that the lower limit of $T_{i}$ for line $j$ is zero. The interval defined by equation (4) is not to be considered the "error bar" due to experimental uncertainties in the measurement of the line's FWHM; it indicates the range of equally possible $T_{i}$ values allowed by the observed FWHM value. Uncertainties in the measured $d \lambda$ are propagated to the limit values of $T_{i}$ in equation (4), and widen the range of possible ion temperatures. This method of analysis has the advantage of reducing the assumptions on $T_{i}$ and $v_{\text {nth }}$ to the very minimum. However, it only provides us with an upper limit to $v_{\text {nth }}$.

\section{OBSERVATIONS AND DATA REDUCTION}

\subsection{Observations}

Observations were taken on 1996 November 3, and consist of a full spectral scan encompassing the entire SUMER wavelength range carried out over a polar coronal hole. The center of the slit field of view was $\left(0^{\prime \prime}, 1149^{\prime \prime}\right)$, so that coronal hole plasma was observed at heliocentric distances between 1.03 solar radii $\left(R_{\odot}\right)$ and $1.33 R_{\odot}$. The instrument configuration allows the recording of the solar spectrum in $\simeq 43 \AA$ wide subsections of the 500-1600 $\AA$ spectral range; in the present observation the full SUMER range was observed in 61 subsections, each shifted from the previous one by $\simeq 13 \AA$. This ensured that all lines were observed on the more sensitive $\mathrm{KBr}$-coated portion of the detector at least once. Each spectral subsection was observed with SUMER detector B with $1200 \mathrm{~s}$ exposure time, so that the final effective wavelength range of the dataset we studied is 500-1500 $\AA$. An image of the SUMER field of view superimposed to EIT images is shown in Figure 1 

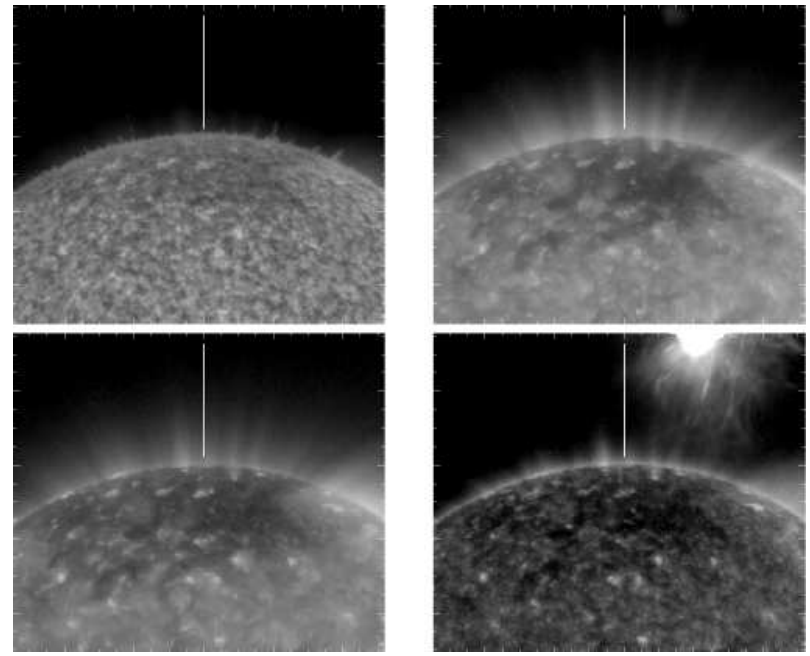

FIG. 1.- Field of view of the SUMER slit, superimposed to EIT images in all four filters: Top left: He II $\left(5 \times 10^{4} \mathrm{~K}\right)$; Top right: Fe IX-X $\left(6.3 \times 10^{5} \mathrm{~K}\right)$; Bottom left: Fe XII $\left(1.4 \times 10^{6} \mathrm{~K}\right)$; Bottom right: Fe XV $\left(2.2 \times 10^{6} \mathrm{~K}\right)$. The EIT images were taken on the same day as SUMER (3 November 1996) between 1 UT and 2 UT.

TABLE 1

LINES USED FOR THE PRESENT STUDY. $R_{\max }$ (IN UNITS OF SOLAR RADII FROM DISK CENTER) INDICATES THE MAXIMUM DISTANCE BEYOND WHICH THE LINE COULD NOT BE USED DUE TO SCATTERED LIGHT CONTAMINATION.

\begin{tabular}{|c|c|c|c|c|c|}
\hline Ion & Wvl. (Å) & $R_{\max }$ & Seq. & $\log T_{\max }$ & $Z_{i} / A_{i}$ \\
\hline Na IX & 681.72 & 1.171 & $\mathrm{Li}$ & 5.92 & 0.348 \\
\hline Si IX & 694.90 & 1.151 & C & 6.05 & 0.285 \\
\hline Ar VIII & 700.25 & 1.100 & $\mathrm{Na}$ & 5.61 & 0.175 \\
\hline Mg IX & 706.06 & 1.171 & $\mathrm{Be}$ & 5.99 & 0.329 \\
\hline Fe VIII & 721.26 & 1.049 & K & 5.88 & 0.125 \\
\hline $\operatorname{Mg} I X$ & 749.55 & 1.171 & $\mathrm{Be}$ & 5.99 & 0.329 \\
\hline Mg VIII & 762.65 & 1.059 & B & 5.91 & 0.288 \\
\hline Mg VIII & 769.38 & 1.079 & B & 5.91 & 0.288 \\
\hline $\mathrm{Ne}$ VIII & 770.42 & 1.171 & $\mathrm{Li}$ & 5.80 & 0.347 \\
\hline Mg VIII & 772.29 & 1.090 & B & 5.91 & 0.288 \\
\hline $\mathrm{Ne}$ VIII & 780.34 & 1.171 & $\mathrm{Li}$ & 5.80 & 0.347 \\
\hline Mg VIII & 782.37 & 1.151 & B & 5.91 & 0.288 \\
\hline Ca IX & 821.22 & 1.049 & $\mathrm{Mg}$ & 5.80 & 0.200 \\
\hline Mg VII & 854.64 & 1.079 & $\mathrm{C}$ & 5.80 & 0.247 \\
\hline Mg VII & 868.08 & 1.110 & C & 5.80 & 0.247 \\
\hline $\mathrm{Ne}$ VII & 887.24 & 1.028 & $\mathrm{Be}$ & 5.71 & 0.297 \\
\hline $\mathrm{Ne}$ VII & 895.16 & 1.100 & $\mathrm{Be}$ & 5.71 & 0.297 \\
\hline S VI & 933.41 & 1.079 & $\mathrm{Na}$ & 5.38 & 0.156 \\
\hline Ne VII & 973.33 & 1.028 & $\mathrm{Be}$ & 5.71 & 0.297 \\
\hline $\mathrm{Fe} X$ & 1028.04 & 1.161 & $\mathrm{Cl}$ & 6.08 & 0.161 \\
\hline O VI & 1031.93 & 1.171 & $\mathrm{Li}$ & 5.58 & 0.313 \\
\hline O VI & 1037.63 & 1.171 & $\mathrm{Li}$ & 5.58 & 0.313 \\
\hline Si VII & 1049.26 & 1.079 & $\mathrm{O}$ & 5.79 & 0.214 \\
\hline Al VII & 1053.88 & 1.059 & $\mathrm{~N}$ & 5.79 & 0.222 \\
\hline Al VII & 1056.79 & 1.059 & $\mathrm{~N}$ & 5.79 & 0.222 \\
\hline Al VIII & 1057.86 & 1.069 & C & 5.94 & 0.259 \\
\hline $\mathrm{Ca} X$ & 557.76 & 1.110 & $\mathrm{Na}$ & 5.87 & 0.225 \\
\hline $\mathrm{Ne} \mathrm{VI}$ & 558.59 & 1.028 & B & 5.63 & 0.248 \\
\hline $\mathrm{Ne}$ VI & 562.80 & 1.028 & B & 5.63 & 0.248 \\
\hline $\mathrm{CaX}$ & 574.01 & 1.110 & $\mathrm{Na}$ & 5.87 & 0.225 \\
\hline $\operatorname{Mg} X$ & 609.80 & 1.171 & $\mathrm{Li}$ & 6.04 & 0.370 \\
\hline $\mathrm{NV}$ & 1238.82 & 1.079 & $\mathrm{Li}$ & 5.24 & 0.286 \\
\hline Fe XII & 1242.00 & 1.141 & $\mathrm{P}$ & 6.22 & 0.197 \\
\hline N v & 1242.81 & 1.069 & $\mathrm{Li}$ & 5.24 & 0.286 \\
\hline $\operatorname{Mg} X$ & 624.97 & 1.161 & $\mathrm{Li}$ & 6.04 & 0.370 \\
\hline Si VIII & 1440.51 & 1.028 & $\mathrm{~N}$ & 5.92 & 0.249 \\
\hline Si VIII & 1445.76 & 1.161 & $\mathrm{~N}$ & 5.92 & 0.249 \\
\hline Fe XI & 1467.07 & 1.100 & $\mathrm{~S}$ & 6.14 & 0.179 \\
\hline
\end{tabular}

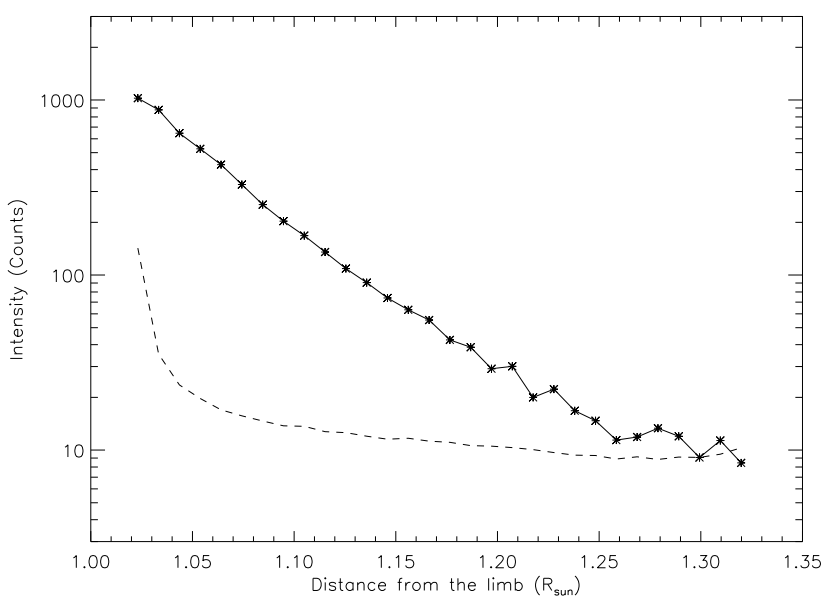

FIG. 2.- Intensity of the Mg IX 706.06 $\AA$ line as a function of distance from the limb (in counts). The scattered light intensity is superimposed as a dashed line, and it has been normalized to match the Mg IX intensity averaged over the three positions farthest from the solar limb.

\subsection{Data reduction and scattered light}

Raw data were reversed and corrected from flat field and geometrical distortions using the standard SUMER software available in the SolarSoft package for IDL. Corrections for deadtime and local gain depression were not necessary because count rates were always sufficiently low. A large set of lines was selected in order to sample as wide a range of $Z_{i} / A_{i}$ ratios as possible with bright, isolated lines that minimized uncertainties tied to line profile fitting. These lines are listed in Table 1. In order to increase the signal-to-noise, the SUMER slit was divided into 10 pixel-wide bins along the slit direction: within each bin, the emission was averaged. The profile of each line was fitted with a Gaussian profile, obtaining line centroid, width and total intensity.

In the present work, we are primarily interested in line widths, but line intensities were used to determine the amount of scattered light contamination. Instrument-scattered light consists of disk radiation scattered by mirror microroughness, that forms on the detector a ghost, unshifted spectrum of the solar disk that contaminates true local emission. A significant scattered light blending may alter line profiles in ways that are not easy to assess and correct. Before attempting an analysis of line widths, it is important to check that the scattered light contribution is negligible and, if not negligible, to remove it. Scattered light can be distinguished from true coronal emission by considering the variations of the measured intensity with distance from the limb, because true emission decreases much faster than instrument-scattered light. In order to evaluate the amount of scattered light contaminating each line, we first determined the rate of decrease of scattered light intensity with distance from the limb. This was done first by measuring the normalized intensity versus height relationship (henceforth scattered light profile) of a few chromospheric and transition region lines which, outside the solar disk, are almost entirely made of scattered light. We then assumed that the emission of the selected coronal lines measured at the farthest distance from the limb is entirely composed of scattered light, and used the scattered light profile to evaluate the absolute intensity of instrument-scattered emission for each coronal line at all heights. An example of comparison between scattered light and true coronal emission is given in Figure 2 for the Mg IX line at $706.06 \AA$. It is impor- 
tant to note that this method actually overestimates the scattered light emission. We considered the scattered light to be negligible when its intensity, as estimated using this method, is $25 \%$ or less of the measured intensity of a coronal line.

This method allowed us to determine the maximum height $R_{\max }$ below which the scattered light can be safely considered negligible. For the strongest lines, we could stretch our measurements up to $1.17 R_{\odot}$, while only the closest bin provided intensity for the weakest lines. Table 1 also reports the value of $R_{\max }$ for each line.

The presence of scattered light in line widths can also be checked independently by considering the quantity $d \lambda / \lambda_{0}$. In fact, from equation 11 it is easy to see that $d \lambda / \lambda_{0}$ only depends on physical properties of the ion and so it is the same for all lines of the same ion, unless some problem affects the profile of a line. We calculated this quantity for all the ions in Table 1 with more than one line in order to check whether there was agreement at all heights up to $R_{\max }$. In the few cases where $d \lambda / \lambda_{0}$ was not constant within the same ion, we have investigated the cause of the disagreement, usually due to poor signal-to-noise or to blending lines and rejected the positions where there were problems. It is important to note that ions with only one line are considered less reliable because their width $d \lambda$ could not be checked using this method.

\section{ION TEMPERATURES}

The ion temperatures measured from line widths are shown in Figure 3, which displays them as a function of the $Z_{i} / A_{i}$ ratio for a few heights above the limb. The analysis method provides rather wide ranges of temperature for almost all ions, so that any height dependence is nearly washed away and the results are qualitatively the same up to the maximum height $1.17 R_{\odot}$. The ions emitting weak lines only provide a few measurements close to the limb, and in all cases they are compatible with a constant $T_{i}$ value even if their $T_{i}$ ranges seem to decrease with height. Brighter lines provide $T_{i}$ measurements up to $1.17 R_{\odot}$, that sometimes seem to increase and sometimes seem to decrease with height; in all cases they too are compatible with constant $T_{i}$. As height increases, fewer and fewer lines are useful to measure $T_{i}$, so that it is more difficult to sample the $Z_{i} / A_{i}$ range as accurately as with observations closer to the limb.

Landi (2008) measured the electron temperature of the same emitting plasma under consideration in the present work, finding that it is nearly isothermal at each height, with temperature slightly increasing with height from $\log \left(T_{e} / \mathrm{K}\right) \simeq$ 5.90 to $\log \left(T_{e} / \mathrm{K}\right) \simeq 6.05$. Figure 3 shows the measured upper and lower limits of $T_{e}$ on top of the $T_{i}$ ranges: in all cases the latter are larger than the former and if agreement is found, it is only because the $T_{i}$ ranges are rather broad. This behavior is apparent even at the lowest heights and it confirms the earlier results by $\mathrm{Tu}$ et al. (1998): coronal hole plasmas are not in equilibrium.

Figure 3 shows that there is a relationship between $T_{i}$ and the $Z_{i} / A_{i}$ ratio. At larger heights the number of available ions is limited so the relationship between $T_{i}$ and $Z_{i} / A_{i}$ is less apparent, or it can not even be determined, although results are consistent with those at lower heights. Figure 3 shows an Ushaped $T_{i}$ versus $Z_{i} / A_{i}$ relationship, where the ions providing large $T_{i}$ at $Z_{i} / A_{i} \leq 0.2$ are Fe X to Fe XII and ArVIII. For $Z_{i} / A_{i}>0.2, T_{i}$ values are more constant, showing only a mild increase with $Z_{i} / A_{i}$. It is interesting to note that all the ions with $T_{i}$ closer to, or in agreement with, $T_{e}$ all have $Z_{i} / A_{i}$ between 0.2 and 0.3 . The relationship between $T_{i}$ and
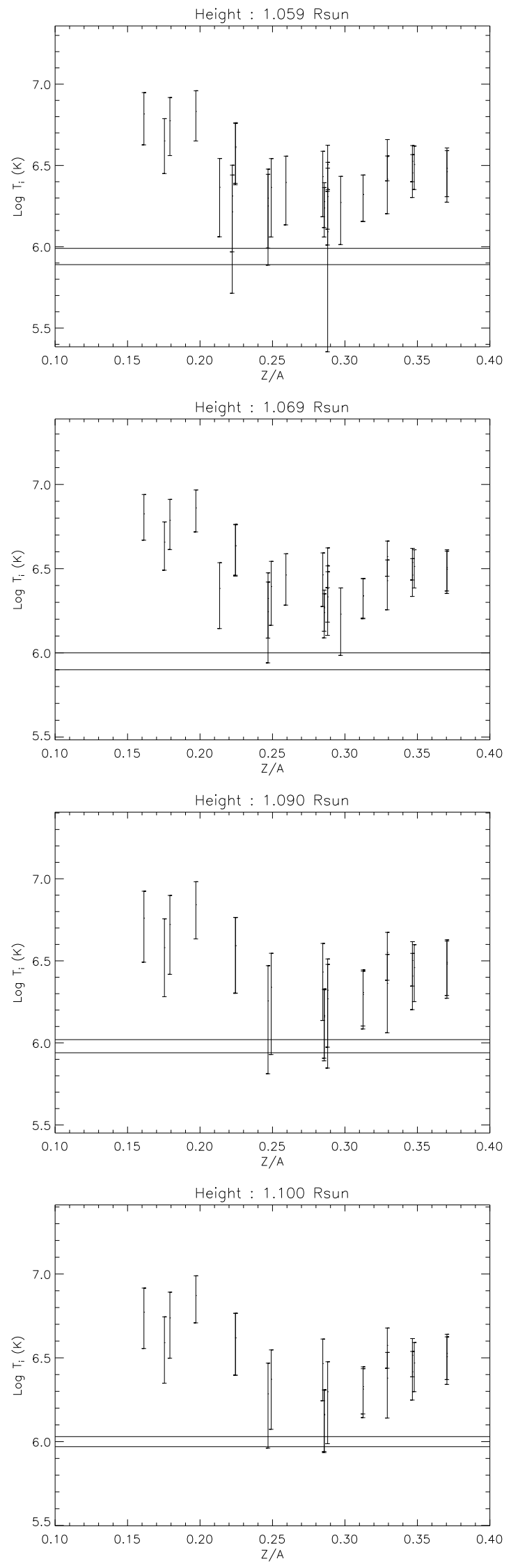

FIG. 3.- Ion temperature versus the charge-over-mass ratio $Z_{i} / A_{i}$, for a selected subset of measured heights. The horizontal lines represent the minimum and maximum value of the electron temperature as measured by Landi (2008) 


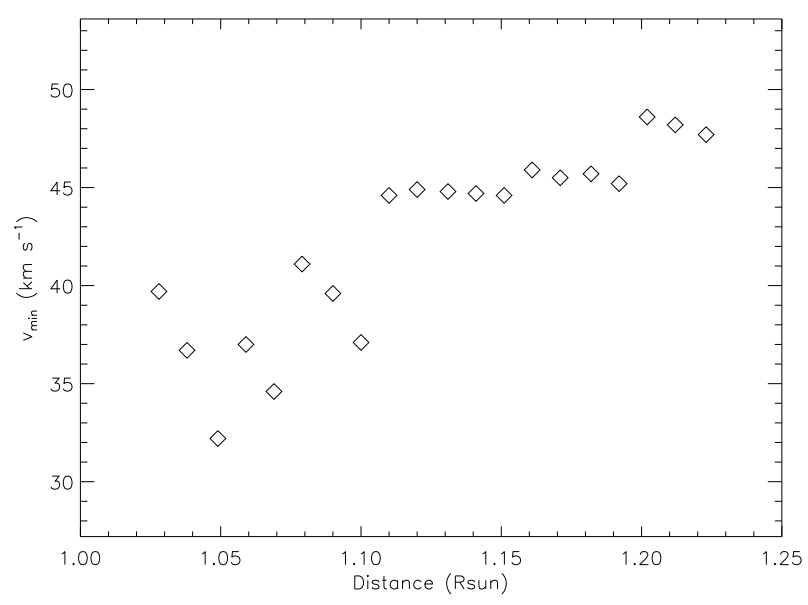

FIG. 4.- Value of $v_{\min }$ as a function of height above the limb.

$Z_{i} / A_{i}$ can be compared with the measurements carried out by Landi (2007) on three different quiet Sun regions using the same diagnostic method. Differences are striking, as quiet Sun $T_{i}$ values show no dependence on the $Z_{i} / A_{i}$ ratio, and they are in much better agreement with the electron temperature than in the present work. However, we found that the lines we selected have a sufficiently large signal-to-noise to provide uncertainties in the limit values of $T_{i}$ that are small when compared to the range of $T_{i}$ given by equation (4).

The Tu et al. (1998) diagnostic technique does not allow us to measure $v_{\mathrm{nth}}$, since equation (3) only allows us to set an upper limit on it. The value of $v_{\min }$ increases only slightly with height from $\simeq 30-35 \mathrm{~km} \mathrm{~s}^{-1}$ to $\simeq 45-50 \mathrm{~km} \mathrm{~s}^{-1}$, and it is shown in Figure 4

\section{THERMAL AND NON-THERMAL COMPONENTS}

As discussed above, the basic assumption in the interpretation of the off-limb line profiles is that there are only two contributors to the (mainly Gaussian) widths: "thermal" microscopic motions and "non-thermal" bulk motions along the line of sight (LOS) due to unresolved waves or turbulence. Another key assumption is that the non-thermal part of the line width has no intrinsic dependence on ion charge or mass. This is valid when the spectrum is dominated by low-frequency MHD waves, but other types of dispersive waves have been suggested to play a role (e.g., Moran 2002; Ofman et al. 2005). However, we retain the standard assumption of no charge or mass dependence of the non-thermal speed, because in most models of MHD turbulence the power that resides at kinetic dispersive scales tends to be much smaller than that at the larger non-dispersive scales.

Figure 5 compares a range of observational and theoretical values for the non-thermal velocity, including the upper limits derived above. This quantity is interpreted as the transverse root mean square Alfvén wave amplitude $\left\langle\delta v_{\perp}^{2}\right\rangle^{1 / 2}$. The wave amplitudes from theoretical models were divided by $\sqrt{2}$ in order to sample only the motions along one of the two perpendicular directions (i.e., only along the LOS). Cranmer \& van Ballegooijen (2005) discussed the observational data of Banerjee et al. (1998) and Esser et al. (1999), as well as other empirical constraints on Alfvén wave properties. The discrepancy between the two theoretical model curves is due to the fact that the Cranmer \& van Ballegooijen (2005) model utilized a fixed empirical set of background plasma parameters

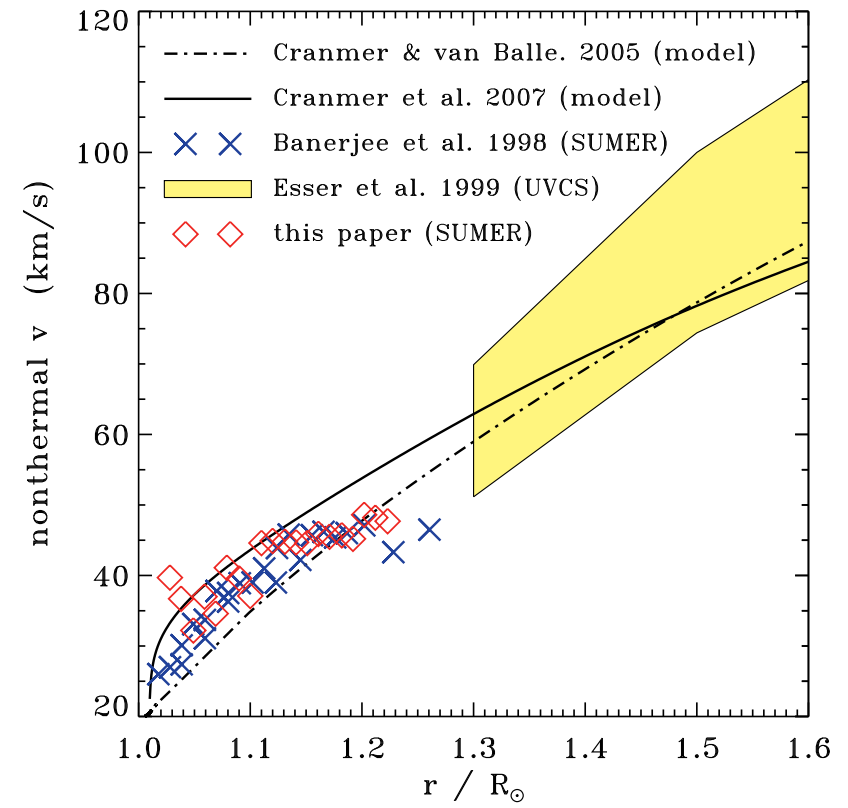

FIG. 5.- Assembled non-thermal velocities, interpreted as perpendicular Alfvén wave amplitudes and plotted versus heliocentric distance in units of the solar radius, from empirical non-WKB wave models (dot-dashed line; Cranmer \& van Ballegooijen 2005) and turbulence-driven coronal heating models (solid line; Cranmer et al. 2007). Also, empirical measurements from Banerjee et al. (1998) (blue crosses), Esser et al. (1999) (yellow region), and from this paper (red diamonds).

(e.g., wind speed, Alfvén speed, density) and Cranmer et al. (2007) computed these properties self-consistently with the waves.

Because in coronal holes (close to the limb) the magnetic field appears to be mainly pointed in the radial direction, the LOS component of the thermal temperature (for ion $i$ ) is assumed to be identical to the perpendicular temperature $T_{\perp i}$. This assumption was validated by test calculations that took account of the full LOS integration and used anisotropic biMaxwellian distributions. These calculations showed that the dominant contribution to a collisional line profile (i.e., sensitive to density squared) comes from a very narrow range of heights, all close enough to the plane of the sky that the sampled component of the distribution always remained close to $T_{\perp i}$.

The model calculations below mainly utilize the ion temperature data at a single reference radius. This radius was chosen as $1.069 R_{\odot}$, which seemed to be the best balance between having a large number of available ions (which drops off as height increases) and the existence of significant "preferential heating" away from thermal equilibrium (which is lessened as height decreases). The measured maximum non-thermal speed at this height is $34.6 \mathrm{~km} \mathrm{~s}^{-1}$, which falls between the modeled values of about $30 \mathrm{~km} \mathrm{~s}^{-1}$ (Cranmer \& van Ballegooijen 2005) and $40 \mathrm{~km} \mathrm{~s}^{-1}$ (Cranmer et al. 2007). However, the actual non-thermal speed must be less than the upper limit derived above. Somewhat arbitrarily, we choose $25 \mathrm{~km} \mathrm{~s}^{-1}$ as a fiducial non-thermal speed for this height, which is used to subtract from the total line width to obtain the individual ion temperatures $T_{\perp i}{ }^{2}$

\footnotetext{
${ }^{2}$ To some extent, when comparing the ion temperatures to one another at the same height, the assumed value for the non-thermal wave amplitude is not so important. As long as the non-thermal speed is independent of ion charge and mass, the relative differences between the various ion temperatures are unchanged.
} 


\section{PHYSICAL PROCESSES IN THE MODEL}

The basic idea of the semi-empirical models to be discussed below is that we assume a turbulent replenishment of ion cyclotron wave power in the corona with a known radial dependence. The absolute normalization for this wave power, though, is determined for a given ion of charge $Z_{i}$ and mass $A_{i}$ iteratively by finding the value that produces the observed level of perpendicular heating at the reference radius of 1.069 $R_{\odot}$. By determining this wave power for the ions, each independently of the others, we construct a model of the overall frequency dependence of the ion cyclotron power spectrum.

The solution for the radial dependence of $T_{\perp i}$ uses an internal energy conservation equation similar to that used by Cranmer et al. (1999a) with some minor changes to be discussed below. The properties of the background proton-electron plasma are held fixed at values determined by comparison with empirical measurements (mainly those of Cranmer \& van Ballegooijen 2005). The heavy ions are thus treated as "test particles" that do not influence the primary plasma or one another. A dominant effect that needs to be included is the temperature equilibration due to Coulomb collisions between protons and the heavy ion species of interest. The high densities in the low corona give rise to rapid collisions that can mask the preferential ion heating from ion cyclotron waves (by driving $T_{\perp i}$ back down to the proton temperature $T_{p}$ ).

The time-steady energy conservation equation that we solve is given by

$$
\frac{d T_{\perp i}}{d r}+\frac{T_{\perp i}}{\mathcal{A}} \frac{d \mathcal{A}}{d r}=\frac{Q_{\perp i}+C_{\perp i p}\left(T_{\perp p}-T_{\perp i}\right)}{n_{i} u_{i} k_{\mathrm{B}}}
$$

where the two terms on the right-hand side represent resonant heating $\left(Q_{\perp i}\right)$ and Coulomb collisions with protons $\left(C_{\perp i p}\right)$. The second term on the left-hand side accounts for adiabatic expansion, and depends on the radial variation in the crosssectional area $\mathcal{A}$ of the polar flux tube. One notable departure from the models of Cranmer et al. (1999a) is that we do not consider the corresponding equation for the parallel ion temperature $T_{\| i}$. Thus, for simplicity, we assume that there is no significant dependence of $T_{\perp i}$ on $T_{\| i}$. Even for strong anisotropy, the isotropization (coupling) term $J_{\perp i p}$ from Cranmer et al. (1999a) is a factor of $\sim 0.2 / A_{i}$ weaker than the standard proton-ion collision term, and thus is negligible.

The proton-ion collision coefficient in equation (5) is given by $C_{\perp i p}=2 k_{\mathrm{B}} n_{i} \nu_{i p}$, where $n_{i}$ is the ion number density. The Coulomb collision rate itself is

$$
\nu_{i p}=\frac{16}{3} \pi^{1 / 2} \ln \Lambda \frac{q_{i}^{2} q_{p}^{2} n_{p}}{m_{i} m_{p} a_{\|} a_{\perp}^{2}}
$$

(e.g., Spitzer 1962), and

$$
\begin{aligned}
& a_{\|} \equiv\left[\left(2 k_{\mathrm{B}} T_{\| i} / m_{i}\right)+\left(2 k_{\mathrm{B}} T_{\| p} / m_{p}\right)\right]^{1 / 2} \\
& a_{\perp} \equiv\left[\left(2 k_{\mathrm{B}} T_{\perp i} / m_{i}\right)+\left(2 k_{\mathrm{B}} T_{\perp p} / m_{p}\right)\right]^{1 / 2}
\end{aligned}
$$

(see also Barakat \& Schunk 1981, 1982; Isenberg 1984). As in Cranmer et al. (1999a), we take the Coulomb logarithm to be a constant of $\ln \Lambda=21$. We assume the protons are isotropic, and we also set $T_{\| i}=T_{p}$ in the definition of $a_{\|}$ (which was verified to hold at the SUMER heights in trial runs using the original equations from Cranmer et al. 1999a). We do not consider collisional interactions between ion species $i$ and any other heavy ion species, which implies that ion $i$ does not ionize or recombine to any other charge states over the range of heights (see, e.g., Lie-Svendsen \& Esser 2005).
The resonant cyclotron heating rate is given in the so-called "optically thin" (narrow resonance zone) limit of Cranmer (2000) as

$$
\frac{Q_{\perp i}}{m_{i} n_{i}}=\frac{4 \pi^{2} \mathcal{P}\left(\Omega_{i}, r\right)}{B_{0}^{2}} \Omega_{i}^{2} V_{\mathrm{A}}^{2}\left(1-\frac{Z_{i}}{A_{i}}\right)
$$

where $B_{0}$ is the radial background magnetic field strength, $\Omega_{i}$ is the ion gyrofrequency, and $V_{\mathrm{A}}$ is the Alfvén speed. The resonant wave power spectrum $\mathcal{P}(\omega)$ differs from the power spectrum used by Cranmer et al. (1999a) by a factor of $4 \pi$ because the latter was defined as the spectrum of the total magnetic fluctuation variance $\left\langle\delta B_{\perp}^{2}\right\rangle . \mathcal{P}(\omega)$, however, is defined as the spectrum of the total fluctuation energy density (see eqs. [15] and [22] below).

Cranmer et al. (1999a) assumed that the power spectrum scaled with the overall radial dependence of $\left\langle\delta B_{\perp}^{2}\right\rangle$, as computed via MHD wave action conservation. However, the relatively small amount of energy in the high-frequency tail of the spectrum may not necessarily scale with the total integrated power. Thus, here we modify this radial dependence using the MHD turbulence theory of Cranmer \& van Ballegooijen (2003) to take account of an assumed anisotropic cascade that maintains the high-frequency tail. We assume that $\mathcal{P}\left(\Omega_{i}, r\right)$ is a separable function of radius and frequency (for which $\omega \approx \Omega_{i}$ ). The radial dependence is given by

$$
\mathcal{P}\left(\Omega_{i}, r\right) \propto \frac{V_{\mathrm{A}}^{3 / 2}}{\rho^{1 / 2}\left(u_{p}+V_{\mathrm{A}}\right)^{3}},
$$

where $\rho$ is the mass density. This scaling relation is derived in Section 7below, and the normalization constant to be used is the main adjustable parameter that we modify to produce agreement with the measured values of $T_{\perp i}$ at $1.069 R_{\odot}$.

The background plasma properties are given by the empirical model of Cranmer \& van Ballegooijen (2005). These properties include the polar flux-tube area $\mathcal{A}(r)$, the outflow speeds (for which we assume that the ions are flowing at the same speed as the protons; $u_{i}=u_{p}$ ), and the number densities. The primary plasma number density $\left(n_{p} \approx n_{e}\right)$ is assumed to have the same radial dependence as the ion number density $n_{i}$. Note that the absolute value of the ion number density is never needed, only its radial dependence.

The Cranmer \& van Ballegooijen (2005) model was essentially a "cold plasma" treatment, so our description of the background proton temperature $T_{p}$ is given by scaling the selfconsistent polar coronal hole temperature of Cranmer et al. (2007) up and down by arbitrary constant factors. The location of the transition region was also scaled down to a standard height of $0.003 R_{\odot}$ above the photosphere. We assume isotropic protons with $T_{\| p}=T_{\perp p} \equiv T_{p}$. The actual proton temperature in the corona is not well constrained by observations, since line ratio techniques are sensitive to the electron temperature, and the widths of $\mathrm{H}^{0}$ lines are more strongly "contaminated" by non-thermal motions than the lines of heavy ions.

In order to determine what to use for the proton temperature in the models, Figure 6 shows a range of measurements and model predictions for $T_{p}$, as well as the electron temperature $T_{e}$ (which is expected to be collisionally coupled to the protons at high enough densities) and the mean one-fluid temperature, which should be approximately proportional to $\left(T_{p}+T_{e}\right) / 2$. Off-limb measurements of $T_{e}$ in polar coronal holes are shown from SUMER data described by Wilhelm (2006) and Landi (2008). Proton temperatures at large heights have been measured by UVCS/SOHO, and the plotted points 


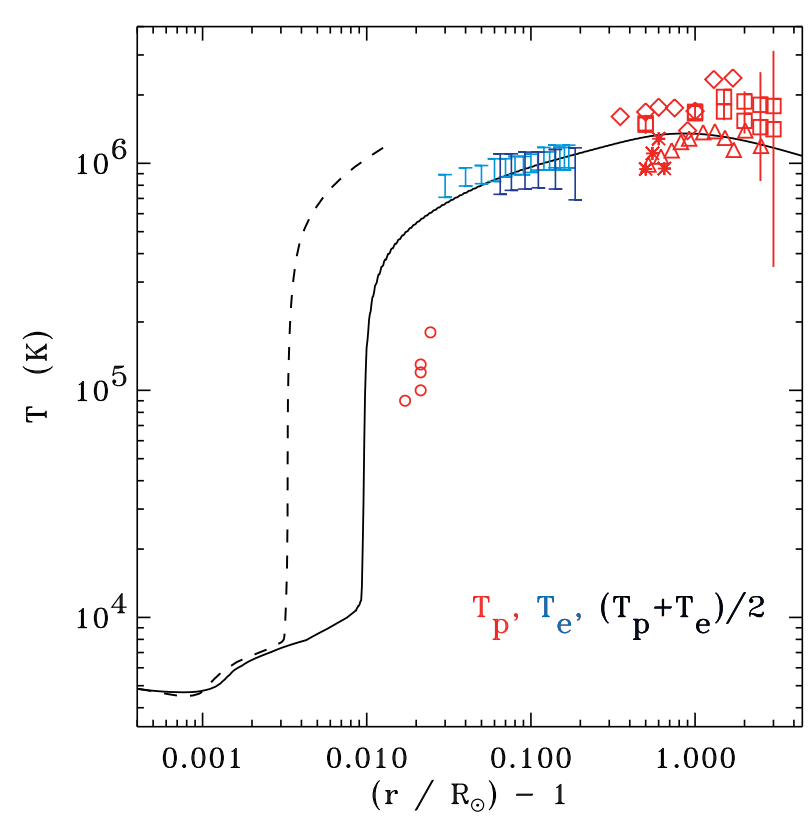

FIG. 6.- Assembled temperatures in polar coronal holes: Mean plasma temperatures from a semi-empirical VAL/FAL-type model (dashed line; Avrett \& Loeser 2008) and from a turbulence-driven coronal heating model (solid line: Cranmer et al. 2007). Te from off-limb SUMER measurements made by Wilhelm (2006) (dark blue bars) and Landi (2008) (light blue bars). $T_{p}$ from off-limb SUMER measurements by Marsch et al. (2000) (circles), and UVCS measurements assembled by Cranmer (2004), with data from Cranmer et al. (1999b) (squares), Esser et al. (1999) (diamonds), Zangrilli et al. (1999) (asterisks), and Antonucci et al. (2000) (triangles).

are those assembled by Cranmer (2004), with a model prediction for the non-thermal wave amplitudes subtracted from the empirical kinetic temperatures. The transition-region-like values of $T_{p}$ at low heights were measured by Marsch et al. (2000); these are quite low compared to most time-steady coronal models that place the sharp transition region at a lower height around $\approx 0.003 R_{\odot}$. These observations may have been influenced by the prevalence of narrow and cool spicules above the limb and may not be representative of the coronal footpoints of solar wind streams.

Before proceeding to discuss the solution of equation (5), it is important to make clear the relative strengths of the individual heating and cooling terms in this conservation equation. As an illustration, Figure 7 shows the radial dependence of timescales computed from each term in equation (5) for the standard choice of parameters (and the $\mathrm{O}^{5+}$ ion) discussed in $\S 8$. Given that the terms in this equation have the units of a spatial temperature gradient, a timescale can be constructed by multiplying by the quantity $u_{i} / T_{\perp i}$ and taking the inverse. For example, the timescale corresponding to ion cyclotron heating is given by

$$
\tau_{\text {heat }}=\frac{n_{i} k_{\mathrm{B}} T_{\perp i}}{Q_{\perp i}} .
$$

The timescale for adiabatic cooling $\tau_{\text {adia }}$ is given by using the second term on the left-hand side of equation (5). Two timescales for Coulomb collisions can be constructed by using either the ion temperature (i.e., the final term in eq. [5]) or the proton temperature (the pentultimate term). These generate the collisional timescales $\tau_{\text {coll }, i}$ and $\tau_{\text {coll, }, p}$, respectively. As can be seen from Figure 7 at low heights the most rapid processes are the Coulomb collisions. Above a height of approximately $0.1 R_{\odot}$, the ion cyclotron heating timescale begins to be as rapid as collisions, and the ion temperature $T_{\perp i}$ begins to

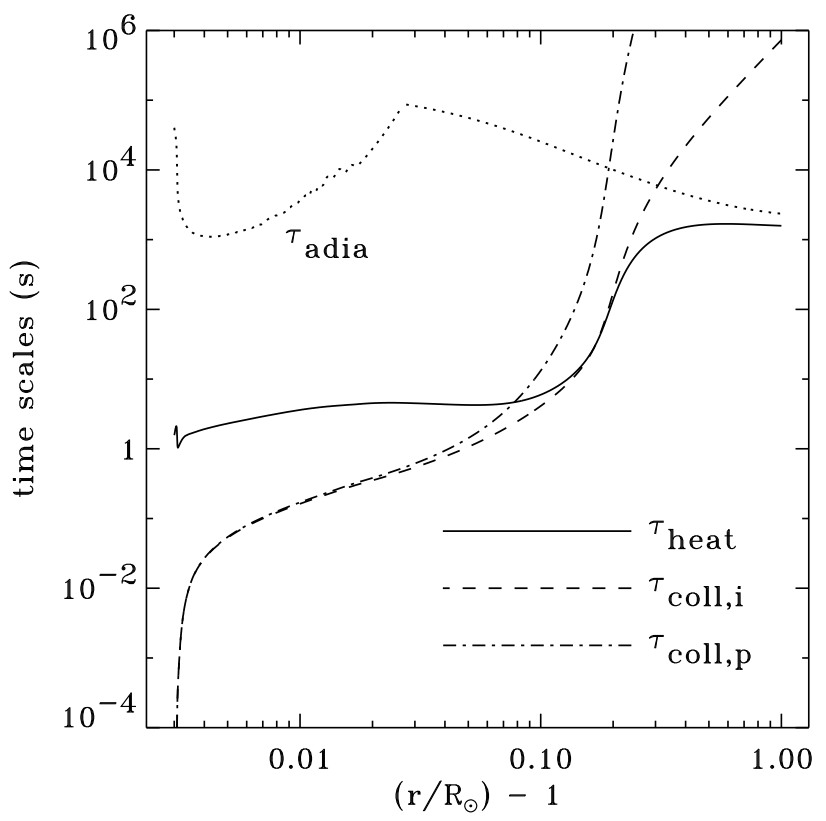

FIG. 7.- Radial dependence of timescales corresponding to individual terms in the energy conservation equation (5); see text for definitions.

increase above the proton temperature. At still larger heights in the extended corona $\left(r \approx 2 R_{\odot}\right)$ adiabatic cooling begins to be as important as ion cyclotron heating, and Coulomb collisions are unimportant.

The numerical integration technique used to compute $T_{\perp i}(r)$ from equation (5) was slightly different from that used by Cranmer et al. (1999a). Because of the strong collisions near the coronal base, the earlier use of simple Euler steps became highly unstable for realistic grid spacings. Instead, we used a piecewise analytic solution of the energy equation that takes account of the collisional coupling explicitly. Equation (5) can be expressed schematically as

$$
\frac{d T_{\perp i}}{d r}+p T_{\perp i}=q
$$

where $p$ and $q$ are considered to be slowly varying functions of radius. The Coulomb collision term has been split up between $p$ and $q$. The analytic solution of this equation, which we use to step the solution for $T_{\perp i}$ up from height $r_{j}$ to height $r_{j+1}$, is given by the integrating factor method as

$$
T_{\perp i, j+1}=\frac{q}{p}+e^{-p\left(r_{j+1}-r_{j}\right)}\left(T_{\perp i, j}-\frac{q}{p}\right) .
$$

In the limit of weak collisions $(p \ll 1)$, this reduces to the standard Euler step,

$$
T_{\perp i, j+1} \approx T_{\perp i, j}+q\left(r_{j+1}-r_{j}\right) .
$$

In the limit of strong collisions (i.e., ignoring the adiabatic expansion and cyclotron heating terms), the ratio $q / p$ reduces to the proton temperature $T_{p}$, and equation (13) is seen to stably drive the solution towards thermal equilibrium.

\section{EXPECTATIONS FROM ANISOTROPIC TURBULENCE THEORY}

As an independent prediction of the resonant wave power, we aim to describe the anisotropic evolution of wave energy in 2D wavenumber space $\left(k_{\|}, k_{\perp}\right)$ by means of the advectiondiffusion theory described by Cranmer \& van Ballegooijen (2003) and in Appendix C of Cranmer \& van Ballegooijen (2005). The theoretical development given in this section is 
somewhat independent of the "semi-empirical" derivation of wave power that comes from solving the equations of the previous section.

The goal is to develop an analytic solution for the turbulent power spectrum (for frequencies near the ion cyclotron resonances) in the limit that the cascade is allowed to proceed to its final "driven" steady state in a small homogeneous volume of plasma. The three-dimensional total power spectrum is defined as

$$
\int d^{3} \mathbf{k} P_{3 \mathrm{D}}(\mathbf{k})=U_{\text {tot }} \approx \frac{\left\langle\delta B_{\perp}^{2}\right\rangle}{4 \pi}
$$

where $U_{\text {tot }}$ is the total energy density of the Alfvén waves, and we write the volume element $d^{3} \mathbf{k}$ in cylindrical coordinates as $2 \pi k_{\perp} d k_{\perp} d k_{\|}$to assume symmetry in the two directions perpendicular to the background field.

For fully developed anisotropic MHD turbulence, we assume the power spectrum to be a separable function of two variables: $k_{\perp}$ and a nonlinearity parameter $y$ defined as the ratio of the local wind-frame frequency $V_{A} k_{\|}$to an assumed nonlinear eddy turnover rate $\langle\delta V\rangle k_{\perp}$ (see, e.g., Goldreich \& Sridhar 1995). We use the notation from Section 2.3 of Cranmer \& van Ballegooijen (2003) and define

$$
P_{3 \mathrm{D}}\left(k_{\|}, k_{\perp}\right)=\frac{\rho V_{A} W_{\perp}^{1 / 2}}{k_{\perp}^{3}} g(y)
$$

where $W_{\perp}\left(k_{\perp}\right)$ is a reduced power spectrum that describes the dominant perpendicular cascade. For the MHD inertial range, the reduced spectrum is given as

$$
W_{\perp}\left(k_{\perp}\right)= \begin{cases}U_{\text {tot }}\left(k_{\perp} / k_{\text {out }}\right)^{-2 / 3}(3 \pi \rho)^{-1}, & k_{\text {out }}<k_{\perp}<k_{\text {in }} . \\ 0, & \text { otherwise }\end{cases}
$$

The above definition assumes the existence of a finite outerscale perpendicular wavenumber $k_{\text {out }}$, which we assumed to be inversely proportional to the correlation length of the turbulence, and an inner-scale wavenumber $k_{\text {in }} \gg k_{\text {out }}$, which we assume to be equivalent to the inverse proton gyrofrequency. The factor of $3 \pi \rho$ above is needed to normalize the full power spectrum as defined in eq. (15).

The $k_{\|}$dependence of the power spectrum is contained in the dimensionless $g(y)$ function given in eq. (16). We define

$$
y=\frac{k_{\|} V_{A}}{k_{\perp} W_{\perp}^{1 / 2}} .
$$

The condition $y=1$ is defined as "critical balance" by Goldreich \& Sridhar (1995), and their analysis only constrains the general shape of $g(y)$, not its exact value. The critical balance condition captures the highly nonlinear state of turbulence, for which a coherent wave survives for no more than about one or two periods before nonlinear processes transfer its energy to the smaller scales. Cranmer \& van Ballegooijen (2003) solved a simple wavenumber diffusion equation to obtain an analytic relation for $g(y)$. For the MHD inertial range, this relation can be shown to be equivalent to

$$
g(y)=\frac{2 \Gamma(n)}{3 \Gamma(n-0.5) \sqrt{\pi}}\left(1+\frac{4 y^{2}}{9}\right)^{-n}
$$

which is normalized to unity when integrated over all $y$, and with

$$
n=1+\frac{3 \beta}{4 \gamma}
$$

The dimensionless constants $\beta$ and $\gamma$ describe the relative strengths of advection and diffusion, respectively, in the $k_{\perp}$ direction. They only occur as the ratio $\beta / \gamma$, which we take to be a free parameter. Cranmer \& van Ballegooijen (2003) discussed the most realistic values for this ratio, which is relatively unconstrained by existing turbulence simulations. The "random walk" turbulence model of van Ballegooijen (1986), though, suggested that $\beta / \gamma \approx 1$. However, Cranmer \& van Ballegooijen (2003) found that one would need this ratio to be smaller than about 0.25 in order to produce enough parallel cascade in the corona to heat protons and heavy ions via cyclotron resonance.

With the above definitions it becomes possible to integrate over $k_{\perp}$ and obtain a reduced 1D power spectrum

$$
P_{1 \mathrm{D}}\left(k_{\|}\right)=2 \pi \int_{k_{\text {out }}}^{k_{\text {in }}} d k_{\perp} k_{\perp} P_{3 \mathrm{D}}\left(k_{\|}, k_{\perp}\right)
$$

which is related to the frequency spectrum $\mathcal{P}(\omega)$ via

$$
P_{1 \mathrm{D}}\left(k_{\|}\right) d k_{\|}=\mathcal{P}(\omega) d \omega .
$$

If we assume, for simplicity, that the ion cyclotron waves of interest are far enough from the proton resonance for the standard MHD dispersion relation $\left(\omega=k_{\|} V_{\mathrm{A}}\right)$ to hold, the above parameterizations for $P_{3 \mathrm{D}}\left(k_{\|}, k_{\perp}\right)$ can be applied to show that

$$
\mathcal{P}(\omega)=\sqrt{\frac{4 \pi \rho U_{\text {tot }}}{3}} k_{\text {out }}^{1 / 3} \int_{k_{\text {out }}}^{k_{\text {in }}} d k_{\perp} \frac{g(y)}{k_{\perp}^{7 / 3}} .
$$

This equation, combined with the cyclotron resonant frequency condition

$$
\omega-u_{i} k_{\|}-\Omega_{i}=0,
$$

is used to produce the "theoretical" power spectra (as a function of the $\beta / \gamma$ parameter) that are compared with the empirically derived power spectra at $1.069 R_{\odot}$. For ion cyclotron waves at any given height in the low corona (where $u_{i} \ll V_{\mathrm{A}}$ ), the wave frequency $\omega$ is directly proportional to the ion charge to mass ratio $Z_{i} / A_{i}$. The main plasma quantities, including the Alfvén wave scale lengths $k_{\text {out }}$ and $k_{\text {in }}$, the total energy density $U_{\text {tot }}$, and the ion Larmor frequencies $\Omega_{i}$, are taken from the radially dependent coronal hole model of Cranmer \& van Ballegooijen (2005).

The above equations specify how the resonant frequency spectrum $\mathcal{P}(\omega)$ can be computed, but it is also helpful to derive a concise relation for the large-scale radial dependence of the wave power in the ion cyclotron frequency range. The gyroresonant wave power exists in the limit of large values of the dimensionless critical-balance parameter $y$. For $y \gg 1$, the function $g(y) \propto y^{-2 n}$, and this implies that the highfrequency tail of the spectrum has a similar power-law dependence $\mathcal{P} \propto \omega^{-2 n}$. Cranmer et al. (1999) treated this exponent as a free parameter $\eta$. Utilizing the definitions given above, this proportionality can be expressed in terms of more basic plasma parameters as

$$
g(y) \propto k_{\perp}^{4 n / 3}\left(\frac{k_{\text {out }}^{2 / 3} U_{\text {tot }}}{3 \pi \rho \Omega_{i}^{2}}\right)^{n} .
$$

Note that the actual frequency dependence is "hidden" in in the ion gyrofrequency $\Omega_{i}$, which is simply $Z_{i} / A_{i}$ times the proton gyrofrequency $\Omega_{p}$. Performing the integration in equation (23) and removing all constants thus provides the radial dependence of the power spectrum:

$$
\mathcal{P} \propto \frac{\rho^{0.5-n} U_{\text {tot }}^{n+0.5} k_{\text {out }}^{(2 n+1) / 3}}{\Omega_{p}^{2(n+2) / 3} w_{p}^{4(n-1) / 3}}
$$




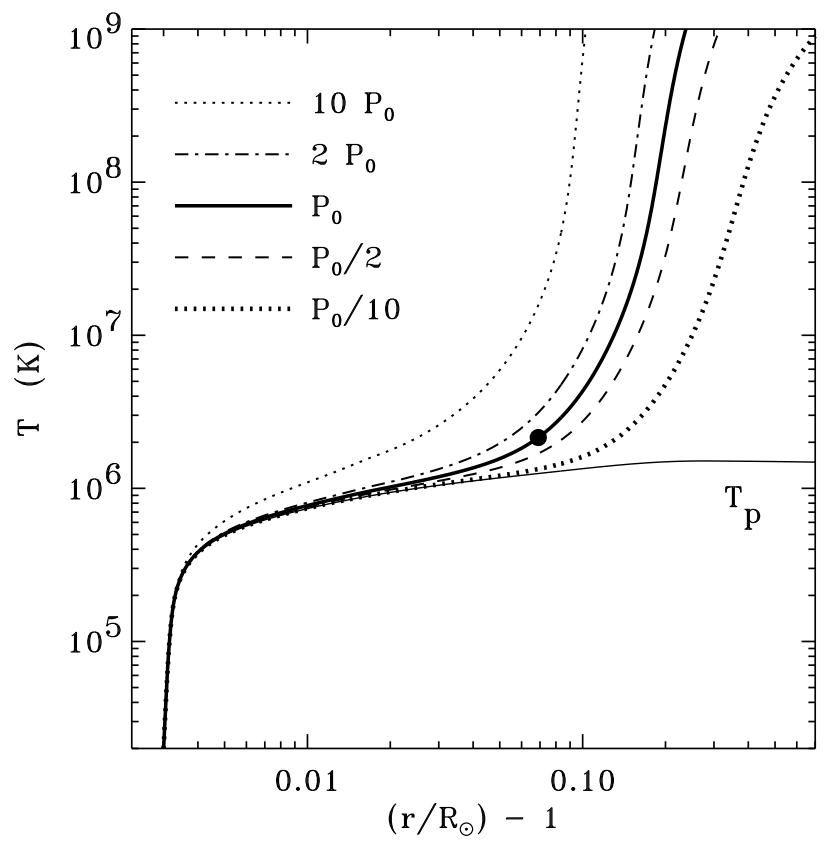

FIG. 8. - Radial dependence of modeled proton temperature (thin solid line) and perpendicular $\mathrm{O}^{5+}$ temperatures, assuming a range of resonant wave power normalization values given relative to $P_{0}$, the iterated value required to heat the ions to the observed level at $1.069 R_{\odot}$ (thick solid line). Resulting values for $T_{\perp i}(r)$ for power levels of $10 P_{0}$ (thin dotted line), $2 P_{0}$ (dot-dashed line), $0.5 P_{0}$ (dashed line), and $0.1 P_{0}$ (thick dotted line) are also shown.

where the proton thermal speed $w_{p}$ is part of the definition of the mean proton gyroradius used in $k_{\text {in }}$. Also, the constant ratio of $Z_{i} / A_{i}$ has been removed in order to isolate the radial dependence for any given ion resonance.

We will see below that the limiting case of $\beta / \gamma \ll 1$ (i.e., $n \approx 1$, or $\eta \approx 2$ in the notation of Cranmer et al. 1999) may be appropriate for comparison with the observations, so let us apply this limit to obtain

$$
\mathcal{P} \propto \rho^{-1 / 2} U_{\text {tot }}^{3 / 2} k_{\text {out }} \Omega_{p}^{-2} .
$$

The outer scale wavenumber $k_{\text {out }}$ is usually assumed to be the reciprocal of the perpendicular correlation length, and the latter is often taken to be proportional to the flux-tube cross section, so we assume $k_{\text {out }} \propto A^{-1 / 2} \propto B_{0}^{1 / 2}$ (Hollweg 1986). We also know that $\Omega_{p} \propto B_{0}$. Finally, the total Alfvén wave energy density can be assumed to scale with the WKB conservation of wave action, which is given by

$$
U_{\text {tot }} \propto \frac{V_{\mathrm{A}}}{\mathcal{A}\left(u_{p}+V_{\mathrm{A}}\right)^{2}} .
$$

Applying these scalings then leads to the radial dependence for the resonant power given in equation (10).

\section{MODEL RESULTS}

The semi-empirical equation of ion energy conservation (eq. [5]) was integrated numerically from the coronal base $\left(1.003 R_{\odot}\right)$ to the reference radius of $1.069 R_{\odot}$ for each of 25 ions having measured SUMER line widths at this height. These ions have $Z_{i} / A_{i}$ values ranging between $0.161\left(\mathrm{Fe}^{9+}\right)$ and $0.370\left(\mathrm{Mg}^{9+}\right)$, and ion temperatures between 1.69 MK $\left(\mathrm{Ne}^{6+}\right)$ and $7.22 \mathrm{MK}\left(\mathrm{Fe}^{11+}\right)$. Also, several grids of models were created for a range of proton temperatures, all with the same relative radial dependence but with values at $1.069 R_{\odot}$ ranging between 0.5 and $3 \mathrm{MK}$.

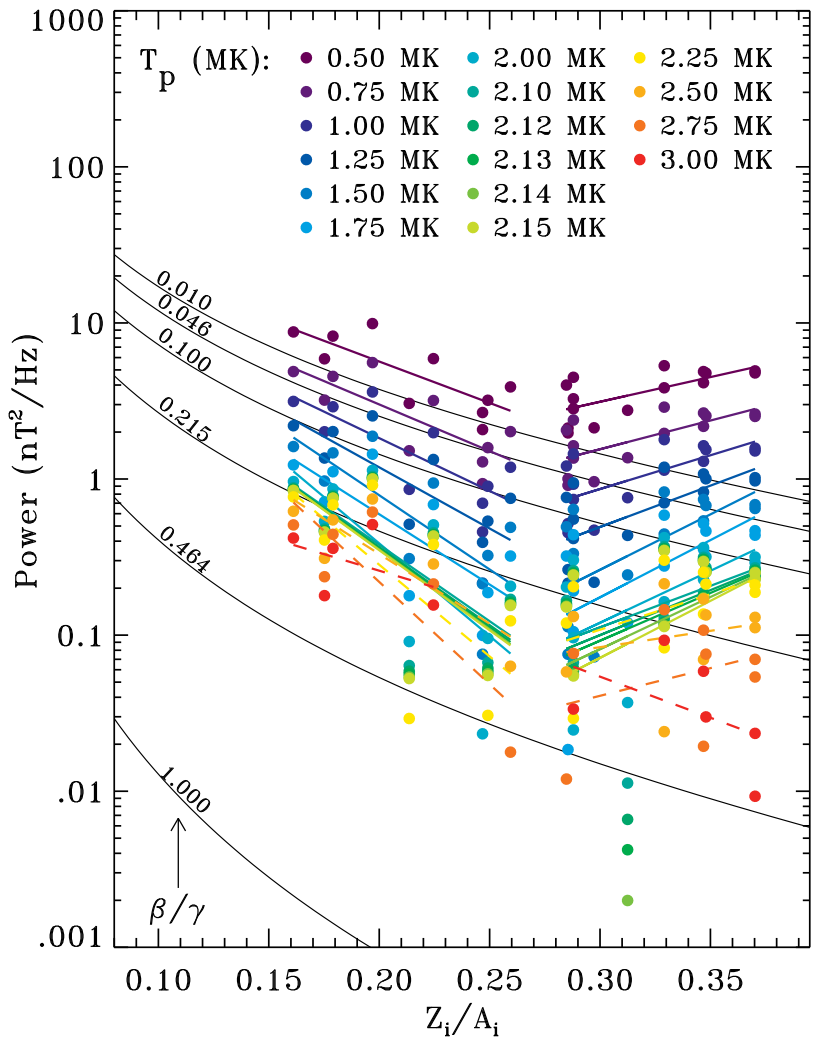

FIG. 9.- Ion cyclotron wave power at $1.069 R_{\odot}$ plotted versus ion chargeto-mass ratio $Z_{i} / A_{i}$ (the latter a proxy for frequency). Empirically determined wave power levels for individual ions (filled circles; see color key for $T_{p}$ normalization) are compared with theoretical predictions for the wave power, based on adopted radially dependent models of the total wave power, Alfvén speed, and other plasma properties (black solid curves).

Figure 8 illustrates the result of this numerical integration for a representative ion $\left(\mathrm{O}^{5+}\right)$ and a specific choice for the proton temperature. The optimal (iterated) wave power necessary to heat these ions to the observed temperature at the reference height is denoted $P_{0}$. Figure 8 also shows the resulting $T_{\perp i}(r)$ curves that are obtained when $P_{0}$ is varied up and down by factors of 2 to 10 . The ion temperature can become several orders of magnitude larger than the proton temperature at larger heights. This has been confirmed by the UVCS $\mathrm{O}$ VI measurements between 1.5 and $3.5 R_{\odot}$ (e.g., Kohl et al. 2006; Cranmer et al. 2008). Note, though, that at such large heights, where Coulomb collisions become very weak, a more proper treatment of the resonant wave-particle interactions is warranted-either via the coupled $T_{\perp i}$ and $T_{\| i}$ equations (Cranmer et al. 1999a) or even a fully kinetic treatment of the ion velocity distributions (e.g., Isenberg \& Vasquez 2007).

Figure 9 shows the result of repeating the numerical integration and wave-power iteration for all 25 ions at $1.069 R_{\odot}$. Recall that no assumptions were made about the frequency dependence of the resonant wave power spectrum for these models. The power for each ion was computed independently of the others. The resonant wave power is shown as a function of $Z_{i} / A_{i}$ for each of the 16 choices for the $T_{p}$ normalization. Straight-line fits are shown for two distinct regions of $Z_{i} / A_{i}$ values, in order to guide the eye and make rough comparisons to the theoretical expectations for $\mathcal{P}(\omega)$. The black curves in Figure 9 are shown to compare the semi-empirical results with the expectations of turbulence theory. In other words, these curves come from evaluating equation (23) numerically for $\mathcal{P}(\omega)$, assuming a range of $\beta / \gamma$ values between 0.01 and 
1.

For large enough choices for $T_{p}$, there occur several ions that have smaller perpendicular temperatures than the adopted proton temperature at $1.069 R_{\odot}$. These are shown with dashed lines for the straight-line fits, and thus should not be considered as self-consistent models for the entire ion data set. In Figure 9, the "green" curves indicate a collection of models all with $T_{p} \approx 2.1 \mathrm{MK}$, because we wanted to explore specifically the behavior of the $\mathrm{O}^{5+}$ heating in the limit of strong collisionality (i.e., because $T_{\perp i} \approx 2.15 \mathrm{MK}$ for this ion); see below.

For the lowest frequencies (i.e., $Z_{i} / A_{i} \lesssim 0.27$ ), both the spectral slope and the absolute wave power at $1.069 R_{\odot}$ seem to agree well with the turbulent cascade predictions for values of $\beta / \gamma \lesssim 0.3$. This agreement is actually quite notable, since the theoretical predictions (black curves) were normalized by pre-existing assumptions about, e.g., the total wave power $U_{\text {tot }}$, and the empirical sets of points were iterated completely independently from any considerations of MHD turbulence. If the proton temperature in the low corona remains less than about 2 MK (see, e.g., Figure 6), then this result provides some additional observational justification for a value of $\beta / \gamma$ of order 0.1 to 0.3 (see Cranmer \& van Ballegooijen 2003).

For the highest frequencies $\left(Z_{i} / A_{i} \gtrsim 0.27\right)$, it seems clear that some kind of "upswing" in the resonant wave power is indicated by the empirical points in Figure9 9 If this is indeed a real effect, there are several possible explanations in terms of MHD and kinetic fluctuations:

1. The additional power at large gyrofrequencies may arise because of plasma instabilities centered around the cyclotron resonances of alpha particles $\left(Z_{i} / A_{i}=0.5\right)$ or protons $\left(Z_{i} / A_{i}=1\right)$; see, e.g., Markovskii (2001), Isenberg (2001), Zhang (2003), Laming (2004), and Markovskii et al. (2006). Thus, the effects seen at charge-to-mass ratios around 0.35 may just be the tail of an additional population of waves that peak at larger frequencies.

2. This may be a similar effect as the spectral flattening (i.e., enhancement above a power law frequency dependence) evident in radio scintillation measurements of density fluctuations at larger heights (e.g., Coles \& Harmon 1989). This flattening was modeled successfully by Harmon \& Coles (2005) by taking into account the enhanced compressibility of obliquely propagating Alfvén waves once they reach ion cyclotron frequencies. It may be possible for compressibility effects to either alter some aspects of the turbulent cascade or change the functional dependence of the heating rate on the available wave power (i.e., eq. [9]) in order to produce this effect.

3. A somewhat more speculative idea is that the particular type of MHD turbulence acting in the low corona may undergo a kind of bottleneck effect where the wave power piles up near the dissipation range. This effect appears in many turbulence simulations, and it has often been suspected of being numerical in origin (e.g., Verma \& Donzis 2007). However, there have been independent theoretical predictions of similar kinds of wave-power pileup arising from nonlocal interactions between disparate scales in $k$-space (e.g., Falkovich 1994; Biskamp et al. 1998).

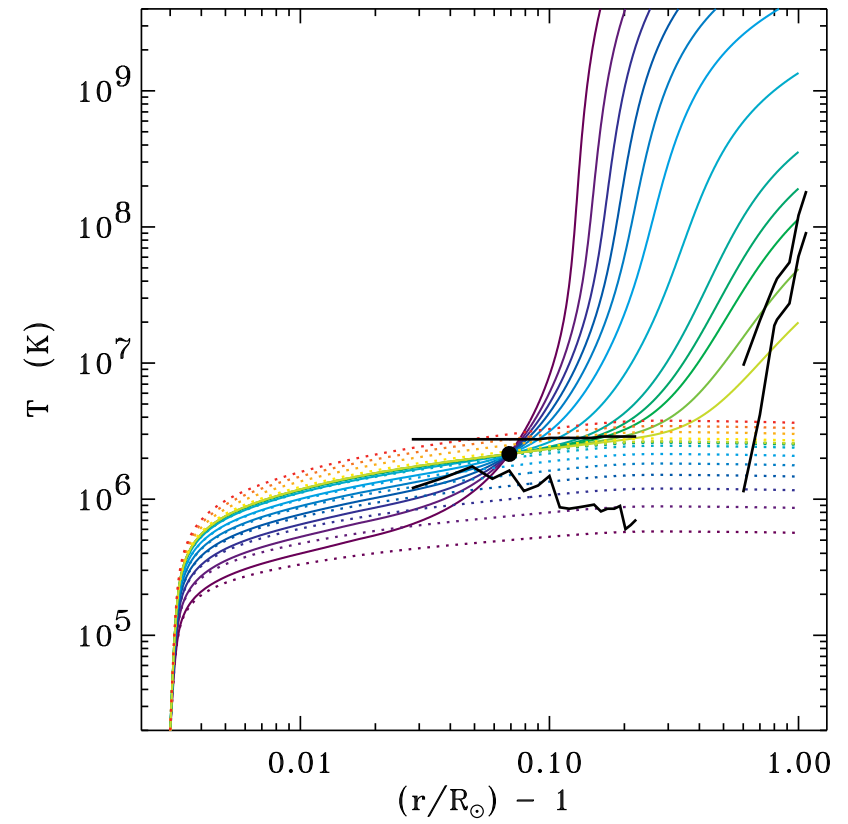

FIG. 10. - Radial dependence of modeled proton temperatures (dotted lines) and perpendicular $\mathrm{O}^{5+}$ temperatures (solid lines), with the same color key as Figure 9 Also shown are observational lower and upper limits on $T_{\perp i}$ from SUMER and UVCS (black solid lines).

Figure 10 illustrates the radial dependence of the ion heating for $\mathrm{O}^{5+}$ ions, and shows how $T_{\perp i}$ varies with the different choices for $T_{p}$. The color scheme for proton temperature values is the same as in Figure 9. The black curves at the heights corresponding to the SUMER measurements show the lower and upper limits defined by equation (4). The black curves at larger heights give the limits on the ion temperature from recent UVCS O VI empirical models (Cranmer et al. 2008).

The models with relatively low proton temperatures $\left(T_{p} \lesssim 2\right.$ $\mathrm{MK})$ require a large amount of resonant wave power to "combat" Coulomb collisions and raise the ion temperature to the observed SUMER value at $1.069 R_{\odot}$. Unfortunately, this large resonant wave power then causes the ion temperature to "explode" to unrealistically huge values at larger heights where the plasma becomes collisionless. On the other hand, the green curves in Figure 10 agree reasonably well with both the SUMER and UVCS data and correspond to larger assumed values for $T_{p}$. Here, the reason for a fine grid of proton temperature curves having

$$
0<\frac{T_{\perp i}}{T_{p}}-1 \ll 1
$$

becomes evident. These choices for $T_{p}$ do not need as much wave power to reach the observed SUMER ion temperature at $1.069 R_{\odot}$, and thus they are less likely to result in unrealistically large temperatures at larger heights. However, it is important to note that these best-fitting solutions seem to have quite strong Coulomb collisions even at the lowest of the SUMER heights. ${ }^{3}$ This would imply that at, say, 1.03$1.05 R_{\odot}$, all of the ion temperatures would be expected to be tightly coupled to both $T_{p}$ and to one another. This, as seen in Figure 3 above, is not the case. Clearly the models presented

\footnotetext{
${ }^{3}$ It is possible, of course, that Coulomb collisions are not the only means of thermalizing and isotropizing the ions. The existence of various kinds of turbulent fluctuations has been suggested to be able to can couple particles and possibly provide extra "quasi-collisions" (e.g., Perkins 1973; Dum 1983; Kellogg 2000). This may need to be investigated in the context of the low corona.
} 
here are only a first step toward a self-consistent description of the preferential ion heating that applies for the full range of heights observed by SUMER and UVCS.

\section{CONCLUSIONS}

In the present work we have used SUMER observations of a polar coronal hole to measure the ion temperatures $T_{i}$ for a large number of ions, in order to determine their dependence on the charge-over-mass ratio $Z_{i} / A_{i}$. We repeated our measurements for several heights between $1.03 R_{\odot}$ and $1.17 R_{\odot}$ in order to investigate the height dependence of our results. We used the method devised by Tu et al. (1998) to determine $T_{i}$; this method only assumed that non-thermal velocities $v_{n t h}$ are the same for all ions.

We found that ion temperatures are larger than the electron temperatures in nearly all cases and at all heights, and that results are qualitatively the same in the $1.03-1.17 R_{\odot}$ range of distances from the limb, although the number of available ions decreases as distance from the limb increases. Our most notable result is an U-shaped dependence of $T_{i}$ from $Z_{i} / A_{i}$, where ions with low $Z_{i} / A_{i}$ (less than about 0.23 ) have very large $T_{i}$ values, while those with $Z_{i} / A_{i} \gtrsim 0.23$ have roughly constant $T_{i}$.

We used our measured $T_{i}$ values to constrain an exploratory model of solar wind heating and acceleration based on ion- cyclotron waves, under the assumption that such waves are gradually replenished as they are dissipated. The measured $T_{i}$ values help us constrain the proton temperatures as well as the ratio between advection and diffusion (in a likely scenario of anisotropic MHD turbulence). We find that the observations of ions having $Z_{i} / A_{i}$ values smaller than about 0.25 are consistent with a turbulence model very similar to that shown by Cranmer \& van Ballegooijen (2003) to be able to energize protons in the extended corona.

However, $T_{i}$ measurements obtained for ions with $Z_{i} / A_{i}$ larger than 0.25 show an upswing in wave power that is difficult to reconcile with traditional views of turbulent cascade. We discuss the implications of this inferred increase in wave power, suggesting several different possibilities such as plasma instabilities, obliquely propagating Alfvén-wave compressibility effects, or a turbulent bottleneck effect.

The work of Enrico Landi is supported by the NNG06EA14I, NNH06CD24C as well as other NASA grants. The work of Steven Cranmer is supported by NASA under grants NNX06AG95G and NNG04GE77G to the Smithsonian Astrophysical Observatory. The authors would like to thank Adriaan van Ballegooijen and Mari Paz Miralles for valuable discussions.

\section{REFERENCES}

Abraham-Shrauner, B., \& Feldman, W. C. 1977, J. Geophys. Res., 82, 618 Antonucci, E., Dodero, M. A., \& Giordano, S. 2000, Sol. Phys., 197, 115 Avrett, E. H., \& Loeser, R. 2008, ApJS, 175, 229

Axford, W. I., \& McKenzie, J. F. 1992, in Solar Wind Seven, ed. E. Marsch \& R. Schwenn (New York: Pergamon), 1

Banerjee, D., Teriaca, L., Doyle, J. G., \& Wilhelm, K. 1998, A\&A, 339, 208 Barakat, A. R., \& Schunk, R. W. 1981, J. Phys. D, 14, 421

Barakat, A. R., \& Schunk, R. W. 1982, Plasma Phys., 24, 389

Biskamp, D., Schwarz, E., \& Celani, A. 1998, Phys. Rev. Lett., 81, 4855

Chandran, B. D. G. 2005, Phys. Rev. Lett., 95, 265004

Coles, W. A., \& Harmon, J. K. 1989, ApJ, 337, 1023

Cranmer, S. R. 2000, ApJ, 532, 1197

Cranmer, S. R. 2001, J. Geophys. Res., 106, 24937

Cranmer, S. R. 2002, Space Sci. Rev., 101, 229

Cranmer, S. R. 2004, in SOHO-15: Coronal Heating, ed. R. W. Walsh, J. Ireland, D. Danesy, \& B. Fleck (Noordwijk, The Netherlands: ESA), ESA SP-575, 154

Cranmer, S. R., Field, G. B., \& Kohl, J. L. 1999a, ApJ, 518, 937

Cranmer, S. R., Panasyuk, A. V., \& Kohl, J. L. 2008, ApJ, 678, 1480

Cranmer, S. R., \& van Ballegooijen, A. A. 2003, ApJ, 594, 573

Cranmer, S. R., \& van Ballegooijen, A. A. 2005, ApJS, 156, 265

Cranmer, S. R., van Ballegooijen, A. A., \& Edgar, R. J. 2007, ApJS, 171, 520

Cranmer, S. R., et al. 1999b, ApJ, 511, 481

Dolla, L., \& Solomon, J. 2008, A\&A, 483, 271

Dum, C. T. 1983, in Solar Wind Five, ed. M. Neugebauer, NASA CP-2280, 369

Esser, R., Fineschi, S., Dobrzycka, D., Habbal, S. R., Edgar, R. J., Raymond, J. C., Kohl, J. L., \& Guhathakurta, M. 1999, ApJ, 510, L63

Falkovich, G. 1994, Phys. Fluids, 6, 1411

Goldreich, P., \& Sridhar, S. 1995, ApJ, 438, 763

Harmon, J. K., \& Coles, W. A. 2005, J. Geophys. Res., 110, A03101

Hollweg, J. V. 1986, J. Geophys. Res., 91, 4111

Hollweg, J. V. 2000, J. Geophys. Res., 105, 7573

Hollweg, J. V., \& Isenberg, P. A. 2002, J. Geophys. Res., 107 (A7), 1147

Hollweg, J. V., \& Turner, J. M. 1978, J. Geophys. Res., 83, 97

Isenberg, P. A. 1984, J. Geophys. Res., 89, 6613
Isenberg, P. A. 2001, J. Geophys. Res., 106, 29249

Isenberg, P. A., \& Hollweg, J. V. 1983, J. Geophys. Res., 88, 3923

Isenberg, P. A., \& Vasquez, B. J. 2007, ApJ, 668, 546

Kellogg, P. J. 2000, ApJ, 528, 480

Kohl, J. L., Noci, G., Cranmer, S. R., \& Raymond, J. C. 2006, A\&A Rev., 13, 31

Laming, J. M. 2004, ApJ, 604, 874

Landi, E. 2007, ApJ, 663, 1363

Landi, E. 2008, ApJ, in press

Lie-Svendsen, Ø., \& Esser, R. 2005, ApJ, 618, 1057

Markovskii, S. A. 2001, ApJ, 557, 337

Markovskii, S. A., Vasquez, B. J., Smith, C. W., \& Hollweg, J. V. 2006, ApJ, 639, 1177

Marsch, E., Goertz, C. K., \& Richter, K. 1982, J. Geophys. Res., 87, 5030

Marsch, E., Tu, C.-Y., \& Wilhelm, K. 2000, A\&A, 359, 381

Moran, T. G. 2002, BAAS, 34, 790 (abstract 88.05)

Ofman, L., Davila, J. M., Nakariakov, V. M., \& Viñas, A.-F. 2005, J. Geophys. Res., 110, A09102

Perkins, F. 1973, ApJ, 179, 637

Ruzmaikin, A., \& Berger, M. A. 1998, A\&A, 337, L9

Seely, J.F., Feldman, U., Schühle, U., Wilhelm, K., Curdt, W., \& Lemaire, P. 1997, ApJ, 484, L87

Spitzer, L., Jr. 1962, Physics of Fully Ionized Gases, 2nd ed. (New York: Wiley)

Tu, C.-Y., \& Marsch, E. 1997, Sol. Phys., 171, 363

Tu, C.-Y., Marsch, E., Wilhelm, K., \& Curdt, W. 1998, ApJ, 503, 475

van Ballegooijen, A. A. 1986, ApJ, 311, 1001

Verma, M. K., \& Donzis, D. 2007, J. Phys. A, 40, 4401

Wilhelm, K. 2006, A\&A, 455, 697

Wilhelm, K., et al. 1995, Sol. Phys., 162, 189

Wilhelm, K., et al. 1997, Sol. Phys., 170, 75

Zangrilli, L., Nicolosi, P., Poletto, G., Noci, G., Romoli, M., \& Kohl, J. L. 1999, A\&A, 342, 592

Zhang, T. X. 2003, ApJ, 597, L69 